SLAC-PUB-11046

BABAR-PROC-04/177

March 3, 2005

\title{
Beyond CP violation: hadronic physics at BaBar
}

\author{
Gabriella Sciolla \\ Massachusetts Institute of Technology, Department of Physics \\ Room 26-443, 77 Massachusetts Avenue, Cambridge, MA \\ (for the BaBar Collaboration)
}

\begin{abstract}
I report on recent studies of hadronic physics performed by the BABAR Collaboration. Emphasis is given to the measurement of the properties of newly discovered charmed hadrons and to the searches for light and heavy pentaquarks.
\end{abstract}

Contributed to the Proceedings of the First APS Topical Group Meeting on Hadron Physics Fermilab, Batavia, IL (October 24-26, 2004).

Stanford Linear Accelerator Center, Stanford University, Stanford, CA 94309

Work supported by Department of Energy contracts DE-FC02-94ER40818 and DE-AC02-76SF00515. 


\section{Introduction}

The BABAR experiment [1] operates at the PEP-II $e^{+} e^{-}$asymmetric B-factory at SLAC. The abundant data sample of over $240 \mathrm{fb}^{-1}$ to date, allows a rich and active research program. The primary goal of the experiment is to perform a quantitative test of the Standard Model, which can be achieved by precise measurements of $\mathrm{CP}$ violation in the $B$ system, and of the CKM matrix elements related to the determination of the sides of the Unitarity Triangle. The study of $B$ decays also provides a sensitive probe for New Physics beyond the Standard Model and allows for tests of Factorization, HQET, HQE and SCET.

In addition to studying $B$ decays, BABAR is also active in the area of spectroscopy of lighter particles. This article focuses on two such topics: charm spectroscopy and searches for pentaquarks.

\section{Recent results in charm spectroscopy}

In 2003 BABAR discovered a narrow resonance [2] in the channel $D_{s}^{+} \pi^{0}$ with a mass of $2317 \mathrm{MeV} / c^{2}$. The existence of this state, named $D_{s J}^{*}(2317)^{+}$, was soon confirmed by CLEO [3] and Belle [4]. Subsequently, the CLEO Collaboration discovered another $D_{s}^{*+} \pi^{0}$ narrow state [3], the $D_{s J}(2460)^{+}$, promptly confirmed by BABAR [4] and Belle [5].

The discovery of the $D_{s J}$ resonances received a lot of attention by the theoretical community and revived the entire field of charm spectroscopy. Many hypotheses were formulated to explain the unexpected narrow widths and masses of these states that are not in agreement with the theoretical predictions. These resonances are usually interpreted as $P$-wave $c \bar{s}$ quark states [6-9], although other interpretations [10-14] cannot be ruled out.

In this section I will report on the improved measurements of the properties of the $D_{s J}^{*}(2317)^{+}$ and $D_{s J}(2460)^{+}[15,16]$. I will also review the recently published evidence for the $X(3872)$ [17] and the status of the search for the $D_{s J}(2632)^{+}[18]$.

\subsection{Mass of the $D_{s J}^{*}(2317)^{+}$and $D_{s J}(2460)^{+}$in $e^{+} e^{-} \rightarrow c \bar{c}$}

BABAR recently published [16] new measurements for the masses of the $D_{s J}^{*}(2317)^{+}$and $D_{s J}(2460)^{+}$. This analysis, based on a dataset of $125 \mathrm{fb}^{-1}$, reconstructs the $D_{s J}$ resonances produced in $c \bar{c}$ events in four final states: $D_{s}^{+} \pi^{0}, D_{s}^{+} \gamma, D_{s}^{+} \pi^{0} \gamma$, and $D_{s}^{+} \pi^{+} \pi^{-}$. The $D_{s}^{+}$candidates are reconstructed in $K^{+} K^{-} \pi^{+}$through $\bar{K}^{* 0} K^{+}$and $\phi \pi^{+}$intermediate states. The $D_{s J}$ candidates are obtained by forming invariant mass combinations of the reconstructed $D_{s}^{+}$mesons with $\pi^{0}, \gamma$, and $\pi^{ \pm}$particles.

Figure 1 (left) shows the invariant mass of the $D_{s J}^{*}(2317)^{+}$candidates reconstructed in the $D_{s}^{+} \pi^{0}$ final state. A clear $D_{s J}^{*}(2317)^{+}$signal is visible on top of the combinatorial background. Reflections from $D_{s J}(2460)^{+} \rightarrow D_{s}^{+} \pi^{0} \gamma$ and $D_{s}^{*}(2112)^{+} \rightarrow D_{s}^{+} \gamma$ are shown in dark and light gray, respectively. A likelihood fit measures a signal yield of $1275 \pm 45$ and an invariant mass of

$$
m\left(D_{s J}^{*}(2317)^{+}\right)=(2318.9 \pm 0.3 \text { (stat.) } \pm 0.9 \text { (syst.) }) \mathrm{MeV} / c^{2} .
$$

Figure 1 (right) shows the $D_{s J}(2460)^{+}$invariant mass in the $D_{s}^{+} \gamma$ final state. The signal is clearly visible on top of a falling combinatorial background. The broader peak centered around $2.3 \mathrm{GeV} / c^{2}$ is a combination of two reflections. The larger reflection is due to $D_{s}^{+}$mesons from the decay $D_{s J}^{*}(2317)^{+} \rightarrow D_{s}^{+} \pi^{0}$ combined with one of the photons from the $\pi^{0}$; the other is produced in a similar fashion from $D_{s J}(2460)^{+} \rightarrow D_{s}^{+} \pi^{0} \gamma$ decay. A signal of $509 \pm 46 D_{s J}(2460)^{+}$candidates 

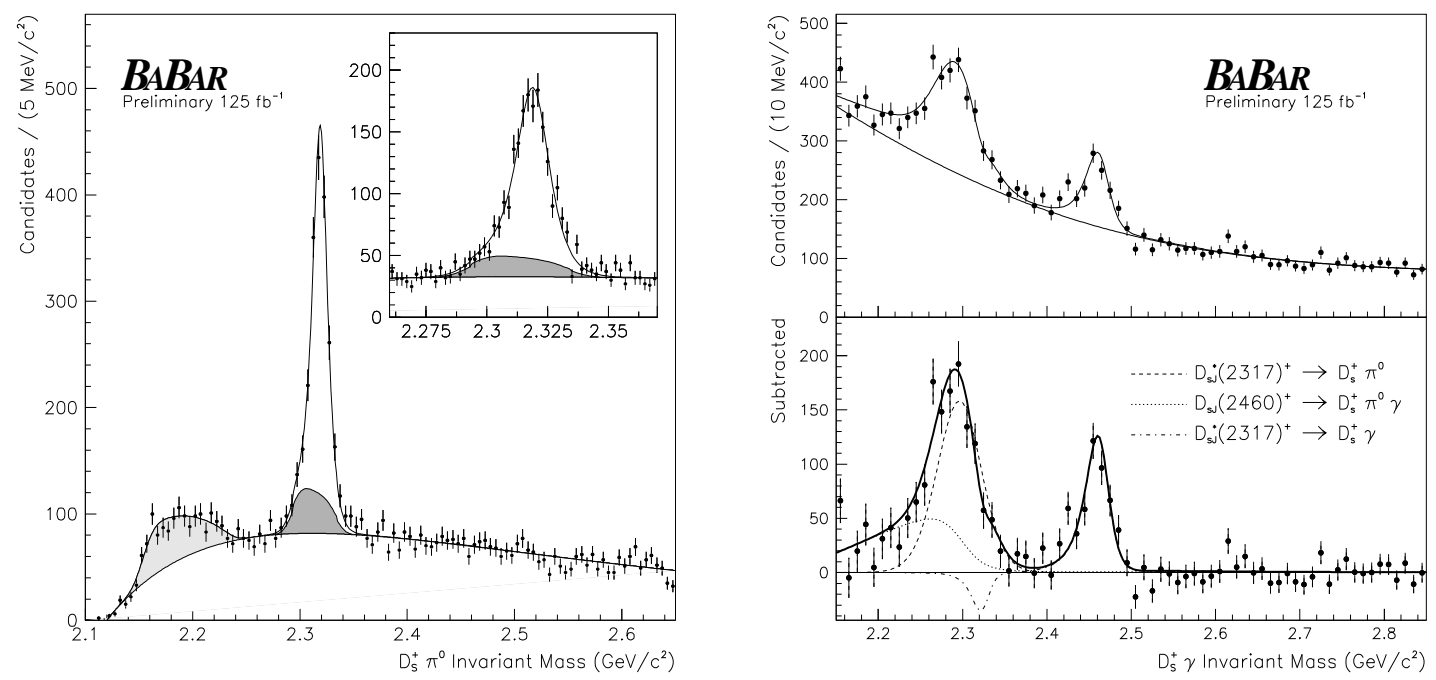

Figure 1: Left: $D_{s}^{+} \pi^{0}$ invariant mass distribution. The solid curve is the result of an unbinned likelihood fit. The dark (light) gray region is the contribution from the $D_{s J}(2460)^{+} \rightarrow D_{s}^{+} \pi^{0} \gamma$ $\left(D_{s}^{*}(2112)^{+} \rightarrow D_{s}^{+} \gamma\right)$ reflection. The inset is an expanded view near the $D_{s J}^{*}(2317)^{+}$mass. Right: $D_{s}^{+} \gamma$ invariant mass distribution before (top) and after (bottom) subtraction of the combinatorial background. The solid curve is the result of an unbinned likelihood fit. Various contributions to the signal and reflection portions of the fit are overlaid (dashed lines).

allows us to measure

$$
\left.m\left(D_{s J}(2460)^{+} \rightarrow D_{s}^{+} \gamma\right)=(2457.2 \pm 1.6 \text { (stat.) } \pm 1.3 \text { (syst. })\right) \mathrm{MeV} / c^{2} .
$$

Figure 2 (left) illustrates the invariant mass distribution for the $D_{s J}(2460)^{+}$decaying into $D_{s}^{+} \pi^{0} \gamma$. This three body decay is dominated by the intermediate state $D_{s}^{*}(2112)^{+} \pi^{0}$. A likelihood fit yields a signal of $292 \pm 29 D_{s J}(2460)^{+}$events. Two reflections are visible in the distribution: the light gray shoulder is due to $D_{s}^{*}(2112)^{+} \rightarrow D_{s}^{+} \gamma$ decays; the dark gray peaking background is due to $D_{s J}^{*}(2317)^{+} \rightarrow D_{s}^{+} \pi^{0}$ decays combined with unassociated photons. The position and shape of the reflections is extracted from Monte Carlo simulation and validated on the data side bands as shown in figure 2 left-top and left-bottom plots. The mass of the $D_{s J}(2460)^{+}$extracted from this measurement is

$$
m\left(D_{s J}(2460)^{+} \rightarrow D_{s}^{+} \pi^{0} \gamma\right)=(2459.1 \pm 1.3 \text { (stat.) } \pm 1.2 \text { (syst.) }) \mathrm{MeV} / c^{2} .
$$

Figure 2 (right) shows the invariant mass of the $D_{s J}(2460)^{+}$candidates reconstructed in the $D_{s}^{+} \pi^{+} \pi^{-}$final state. A signal of $67 \pm 11 D_{s J}(2460)^{+}$decays is clearly visible on top of the combinatorial background; a second peak due to $124 \pm 18 D_{s 1}(2536)^{+}$particles is also evident. The masses for these two particles are extracted by using a likelihood fit:

$$
\begin{aligned}
m\left(D_{s J}(2460)^{+} \rightarrow D_{s}^{+} \pi^{+} \pi^{-}\right) & =(2460.1 \pm 0.3 \text { (stat.) } \pm 1.2 \text { (syst.) }) \mathrm{MeV} / c^{2} \\
m\left(D_{s 1}(2536)^{+}\right) & =(2534.3 \pm 0.4 \text { (stat.) } \pm 1.2 \text { (syst.) }) \mathrm{MeV} / c^{2} .
\end{aligned}
$$



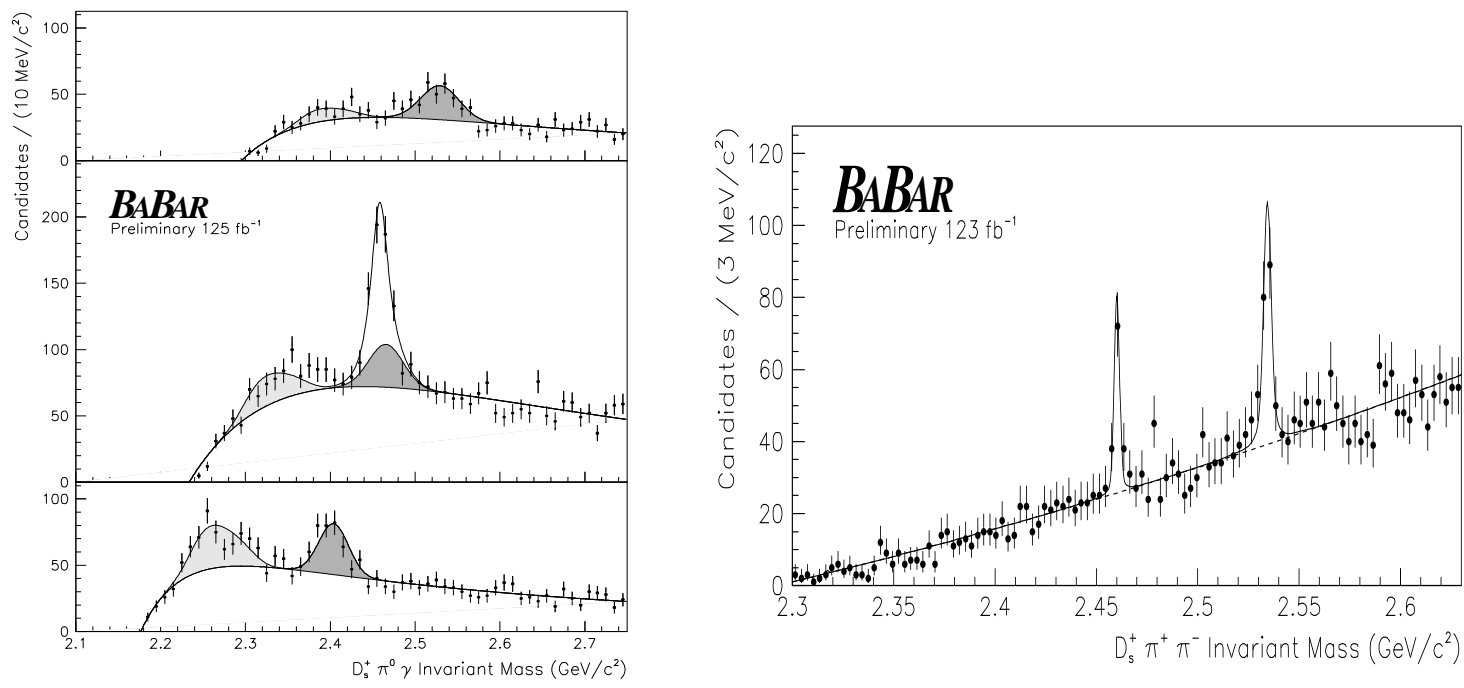

Figure 2: Left: $D_{s}^{+} \pi^{0} \gamma$ mass spectrum of the events that fall in the $D_{s}^{+} \gamma$ signal region (center plot), the $D_{s}^{+} \gamma$ high mass sideband (top plot), and the low mass sideband (bottom plot). Reflections from (dark gray) $D_{s J}^{*}(2317)^{+} \rightarrow D_{s}^{+} \pi^{0}$ and (light gray) $D_{s}^{*}(2112)^{+} \rightarrow D_{s}^{+} \gamma$ decays appear well modeled in the Monte Carlo. Right: $D_{s}^{+} \pi^{+} \pi^{-}$invariant mass distribution for candidates that satisfy the requirements discussed in the text. The solid curve is the result of an unbinned likelihood fit.

The mass measurements mentioned above are combined to obtain the following average:

$$
m\left(D_{s J}(2460)^{+}\right)=(2459.4 \pm 0.3 \text { (stat.) } \pm 1.0 \text { (syst.) }) \mathrm{MeV} / c^{2} .
$$

By combining the efficiency corrected yields, the following relative branching fractions are measured:

$$
\begin{aligned}
\frac{\mathcal{B}\left(D_{s J}(2460)^{+} \rightarrow D_{s}^{+} \gamma\right)}{\mathcal{B}\left(D_{s J}(2460)^{+} \rightarrow D_{s}^{+} \pi^{0} \gamma\right)} & =0.375 \pm 0.054 \text { (stat.) } \pm 0.057 \text { (syst.) } \\
\frac{\mathcal{B}\left(D_{s J}(2460)^{+} \rightarrow D_{s}^{+} \pi^{+} \pi^{-}\right)}{\mathcal{B}\left(D_{s J}(2460)^{+} \rightarrow D_{s}^{+} \pi^{0} \gamma\right)} & =0.082 \pm 0.018 \text { (stat.) } \pm 0.011 \text { (syst.) }
\end{aligned}
$$

\subsection{Properties of $D_{s, J}^{*}(2317)^{+}$and $D_{s J}(2460)^{+}$from exclusive $B$ decays}

Pure samples of $D_{s J}^{*}(2317)^{+}$and $D_{s J}(2460)^{+}$can be obtained by exclusive reconstruction of the decay $B \rightarrow D_{s J}^{(*)+} \bar{D}^{(*)}[15]$. This analysis, performed on a sample of $113 \mathrm{fb}^{-1}$, measures the spin and branching fractions of the $D_{s J}$ resonances.

The $\bar{D}$ and $D_{s}^{+}$mesons are reconstructed in the following decay modes: $\bar{D}^{0} \rightarrow K^{+} \pi^{-}, K^{+} \pi^{-} \pi^{0}$, $K^{+} \pi^{-} \pi^{+} \pi^{-} ; D^{-} \rightarrow K^{+} \pi^{-} \pi^{-} ; D_{s}^{+} \rightarrow \phi \pi^{+}\left(\phi \rightarrow K^{+} K^{-}\right), \bar{K}^{* 0} K^{+}\left(\bar{K}^{* 0} \rightarrow K^{-} \pi^{+}\right)$. The $D^{*}$ candidates are reconstructed in the decay modes $D^{*+} \rightarrow D^{0} \pi^{+}, D^{* 0} \rightarrow D^{0} \pi^{0}, D^{0} \gamma$, and $D_{s}^{*} \rightarrow D_{s}^{+} \gamma$. The selected pairs of $D_{s}^{(*)+}$ and $\bar{D}^{(*)}$ candidates are combined with a photon or a $\pi^{0}$ to complete the $B$ decay. Cuts on the energy and invariant mass of the $B$ candidate are used to enhance the purity of the signal. 

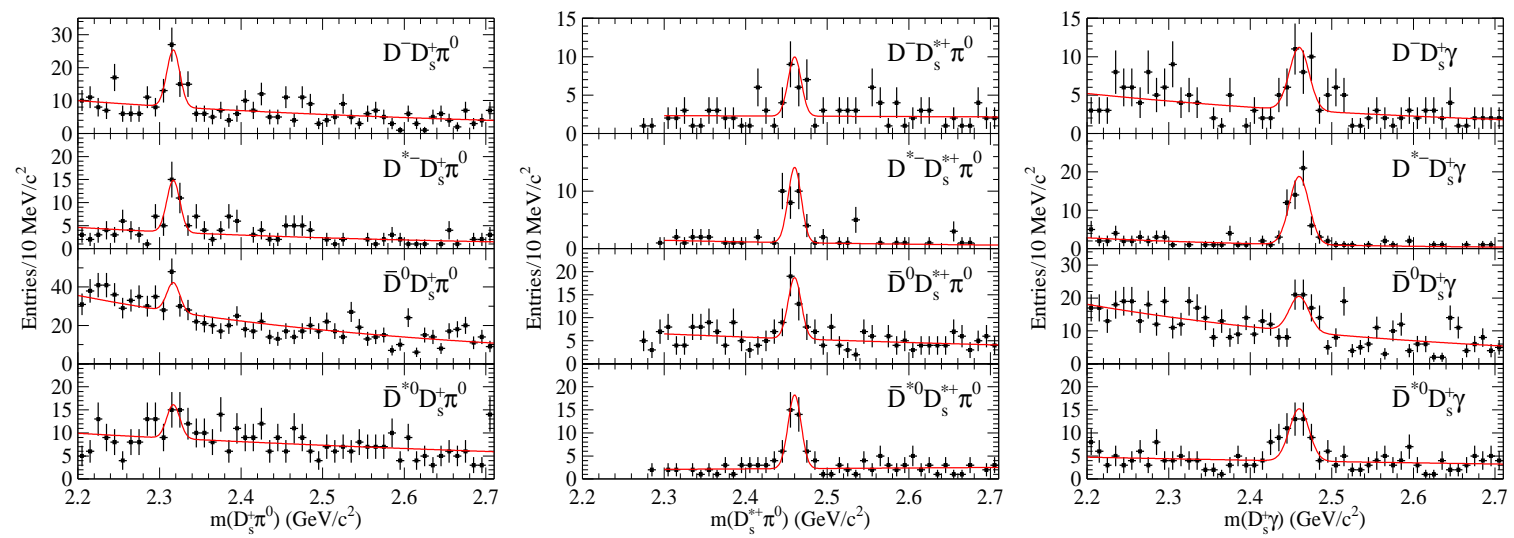

Figure 3: $D_{s}^{+} \pi^{0}$ (left), $D_{s}^{*+} \pi^{0}$ (center), and $D_{s}^{+} \gamma$ (right) mass spectra of the selected $B$ signal candidates for the $12 \bar{D}^{(*)} D_{s J}$ final states. Curves are the results of the fits.

Figure 3 shows the $D_{s J}$ invariant mass for the selected $B$ candidates for 12 different $\bar{D}^{(*)} D_{s J}$ final states. The yields, the corresponding branching fractions and the statistical significance of each channel are listed in Table 1.

From the measured branching fractions for $B \rightarrow D_{s J}^{+}(2460) \bar{D}^{(*)}$ in $D_{s}^{*+} \pi^{0}$ and in $D_{s}^{+} \gamma$, we find the ratio

$$
\frac{\mathcal{B}\left(D_{s J}(2460)^{+} \rightarrow D_{s}^{+} \gamma\right)}{\mathcal{B}\left(D_{s J}(2460)^{+} \rightarrow D_{s}^{*+} \pi^{0}\right)}=0.274 \pm 0.045 \pm 0.020
$$

in agreement with the prediction from [7].

A helicity analysis is performed for the $D_{s J}(2460)^{+}$state, using the decays $B^{+} \rightarrow D_{s J}(2460)^{+} \bar{D}^{0}$ and $B^{0} \rightarrow D_{s J}(2460)^{+} D^{-}$, with $D_{s J}(2460)^{+} \rightarrow D_{s}^{+} \gamma$. The helicity angle $\theta_{h}$ is defined as the angle between the $D_{s J}$ momentum in the $B$-meson rest frame and the $D_{s}$ momentum in the $D_{s J}$ rest frame. Figure 4 shows the resulting angular distribution, corrected for detector acceptance and selection efficiency. The data exclude the $J=2$ hypothesis $\left(\chi^{2} /\right.$ n.d.f. $\left.=36.4 / 4\right)$, while they are in good agreement with the hypothesis of $J=1\left(\chi^{2} /\right.$ n.d.f.=4.0/4). A $J=0$ spin is ruled out by parity and angular momentum conservation in the decay $D_{s J}(2460)^{+} \rightarrow D_{s}^{+} \gamma$.

\subsection{Observation of the $X(3872)$ in $B$ decays}

A new state, known as $X(3872)$, was recently discovered by the Belle Collaboration [19] and confirmed by CDF [20]. This state, reconstructed in the final state $J / \psi \pi^{+} \pi^{-}$, is probably a charmonium candidate for the state $1^{3} D_{2}\left(J^{P C}=2^{--}\right)$or $1^{3} D_{3}\left(J^{P C}=3^{--}\right)$[21], but could also be a molecule of $D$ and $D^{*}$ mesons [22].

BABAR recently confirmed the existence of this particle [17]. The analysis, based on 117 million $B \bar{B}$ events, reconstructs the $X(3872)$ in the final state $J / \psi \pi^{+} \pi^{-}$for $X(3872)$ produced in the decay $B^{-} \rightarrow X(3872) K^{-}$. A total of $30.6 \pm 5.8$ events are reconstructed. The mass of the $X(3872)$ is measured to be $m(X(3872))=(3873.4 \pm 1.4) \mathrm{MeV} / c^{2}$, and the product branching fraction $\mathcal{B}\left(B^{-} \rightarrow X(3872) K^{-}\right) \times \mathcal{B}\left(X(3872) \rightarrow J / \psi \pi^{+} \pi^{-}\right)=(1.28 \pm 0.41) \times 10^{-5}$ is obtained. 
Table 1: Event yields, final branching fractions $\mathcal{B}$, and significance for $B \rightarrow D_{s J}^{(*)+} \bar{D}^{(*)}$ decays. The first error on $\mathcal{B}$ is statistical, the second is systematic, and the third is from the $\bar{D}$ and $D_{s}^{+}$ branching fractions.

\begin{tabular}{llccc}
\hline \hline \multicolumn{1}{c}{$B$ mode } & \multicolumn{2}{c}{ Yield } & $\mathcal{B}\left(10^{-3}\right)$ & Significance \\
\hline$B^{0} \rightarrow D_{s J}^{*}(2317)^{+} D^{-}$ & {$\left[D_{s}^{+} \pi^{0}\right]$} & $34.7 \pm 8.0$ & $1.8 \pm 0.4 \pm 0.3_{-0.4}^{+0.6}$ & 5.5 \\
$B^{0} \rightarrow D_{s J}^{*}(2317)^{+} D^{*-}$ & {$\left[D_{s}^{+} \pi^{0}\right]$} & $23.5 \pm 6.1$ & $1.5 \pm 0.4 \pm 0.2_{-0.3}^{+0.5}$ & 5.2 \\
$B^{+} \rightarrow D_{s J}^{*}(2317)^{+} \bar{D}^{0}$ & {$\left[D_{s}^{+} \pi^{0}\right]$} & $32.7 \pm 10.8$ & $1.0 \pm 0.3 \pm 0.1_{-0.2}^{+0.4}$ & 3.1 \\
$B^{+} \rightarrow D_{s J}^{*}(2317)^{+} \bar{D}^{* 0}$ & {$\left[D_{s}^{+} \pi^{0}\right]$} & $17.6 \pm 6.8$ & $0.9 \pm 0.6 \pm 0.2_{-0.2}^{+0.3}$ & 2.5 \\
$B^{0} \rightarrow D_{s J}(2460)^{+} D^{-}$ & {$\left[D_{s}^{*+} \pi^{0}\right]$} & $17.4 \pm 5.1$ & $2.8 \pm 0.8 \pm 0.5_{-0.6}^{+1.0}$ & 4.2 \\
$B^{0} \rightarrow D_{s J}(2460)^{+} D^{*-}$ & {$\left[D_{s}^{*+} \pi^{0}\right]$} & $26.5 \pm 5.7$ & $5.5 \pm 1.2 \pm 1.0_{-1.2}^{+1.9}$ & 7.4 \\
$B^{+} \rightarrow D_{s J}(2460)^{+} \bar{D}^{0}$ & {$\left[D_{s}^{*+} \pi^{0}\right]$} & $29.0 \pm 6.8$ & $2.7 \pm 0.7 \pm 0.5_{-0.6}^{+0.9}$ & 5.1 \\
$B^{+} \rightarrow D_{s J}(2460)^{+} \bar{D}^{* 0}$ & {$\left[D_{s}^{*+} \pi^{0}\right]$} & $30.5 \pm 6.4$ & $7.6 \pm 1.7 \pm 1.8_{-1.6}^{+2.6}$ & 7.7 \\
$B^{0} \rightarrow D_{s J}(2460)^{+} D^{-}$ & {$\left[D_{s}^{+} \gamma\right]$} & $24.8 \pm 6.5$ & $0.8 \pm 0.2 \pm 0.1_{-0.2}^{+0.3}$ & 5.0 \\
$B^{0} \rightarrow D_{s J}(2460)^{+} D^{*-}$ & {$\left[D_{s}^{+} \gamma\right]$} & $53.0 \pm 7.8$ & $2.3 \pm 0.3 \pm 0.3_{-0.5}^{+0.8}$ & 11.7 \\
$B^{+} \rightarrow D_{s J}(2460)^{+} \bar{D}^{0}$ & {$\left[D_{s}^{+} \gamma\right]$} & $31.9 \pm 9.0$ & $0.6 \pm 0.2 \pm 0.1_{-0.1}^{+0.2}$ & 4.3 \\
$B^{+} \rightarrow D_{s J}(2460)^{+} \bar{D}^{* 0}$ & {$\left[D_{s}^{+} \gamma\right]$} & $34.6 \pm 7.6$ & $1.4 \pm 0.4 \pm 0.3_{-0.3}^{+0.5}$ & 6.0 \\
\hline \hline
\end{tabular}

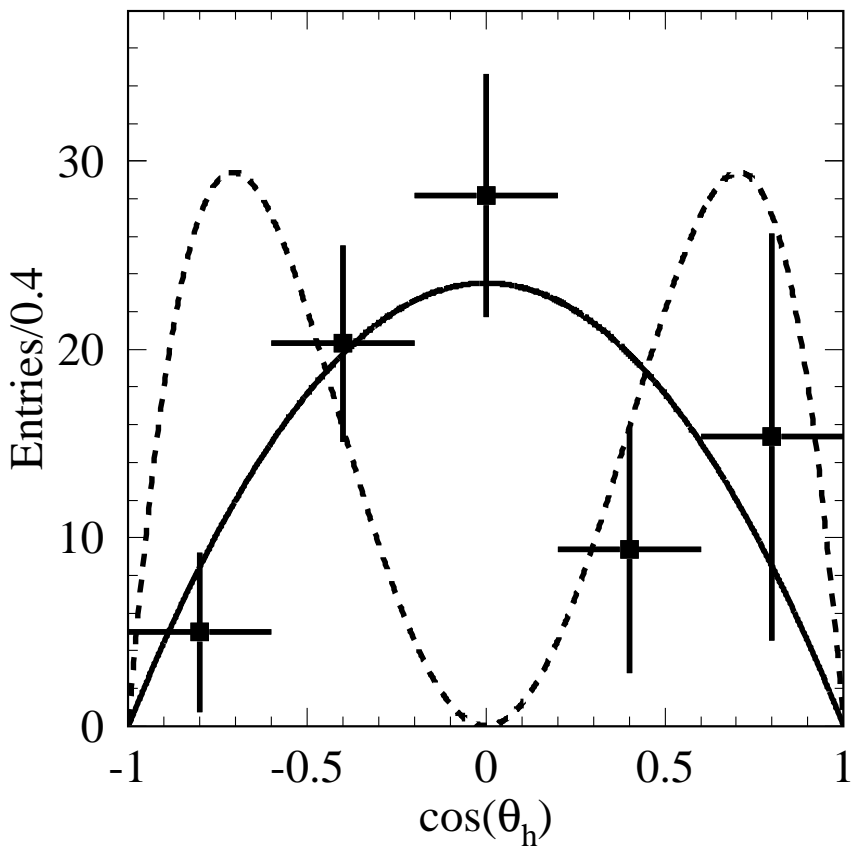

Figure 4: Helicity distribution obtained from $m\left(D_{s} \gamma\right)$ fits in bins of $\cos \left(\theta_{h}\right)$ for data (points) in comparison with the expectations for a $D_{s J}(2460)^{+}$spin $J=1$ (solid line) and $J=2$ (dashed line), respectively, after normalizing the predicted spectra to the data. 

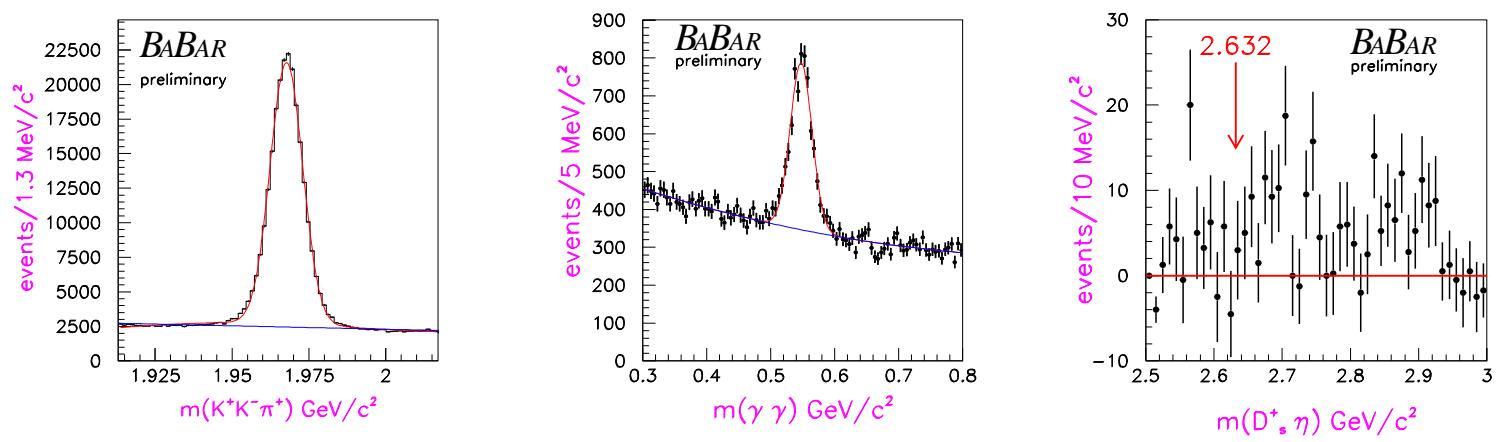

Figure 5: $K^{+} K^{-} \pi^{+}$(left), $\gamma \gamma$ (middle), and $D_{s}^{+} \eta$ (right) invariant mass distributions in $125 \mathrm{fb}^{-1}$.
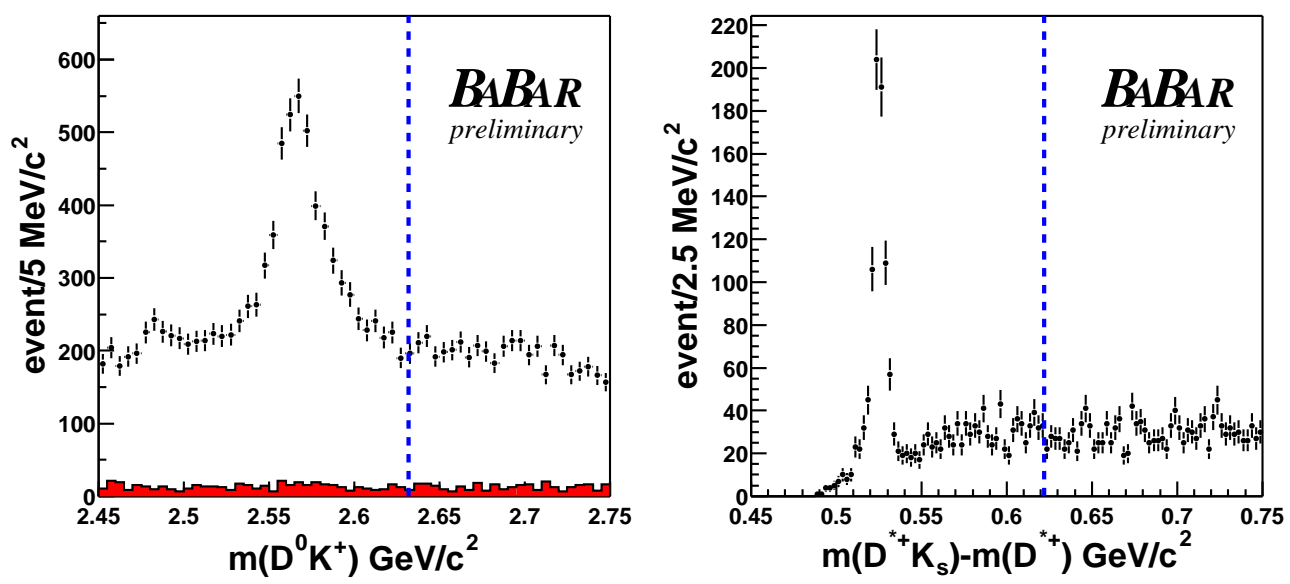

Figure 6: $D^{0} K^{+}$(left) and $D^{*+} K_{S}^{0}$ (right) invariant mass distributions in $125 \mathrm{fb}^{-1}$.

\subsection{Search for the $D_{s J}^{*}(2632)^{+}$meson}

A narrow state decaying to $D_{s}^{+} \eta$, named $D_{s J}^{*}(2632)^{+}$, has been recently observed by the SELEX Collaboration [23]. BABAR has searched for this resonance in the final states $D_{s}^{+} \eta, D^{0} K^{+}$and $D^{*+} K_{S}^{0}$. The analysis, based on $125 \mathrm{fb}^{-1}$, aims to reconstruct $D_{s J}$ states produced in $e^{+} e^{-} \rightarrow c \bar{c}$ events.

Figure 5 shows the abundant and clean samples of $D_{s}^{+} \rightarrow K^{+} K^{-} \pi^{+}$(left) and $\eta \rightarrow \gamma \gamma$ (middle) used for this analysis. The background subtracted invariant mass of the $D_{s}^{+} \eta$ system is shown in figure 5] (right). No signal is evident in the region around $2632 \mathrm{MeV} / c^{2}$. Figure 6] shows the invariant mass of the systems $D^{0} K^{+}$(left) and $D^{*+} K_{S}^{0}$ (right). These distributions show very clear signals for the decays $D_{s 2}(2573)^{+} \rightarrow D^{0} K^{+}$and $D_{s 1}(2536)^{+} \rightarrow D^{*+} K_{S}^{0}$, but no accumulation where the $D_{s J}^{*}(2632)$ is expected. 


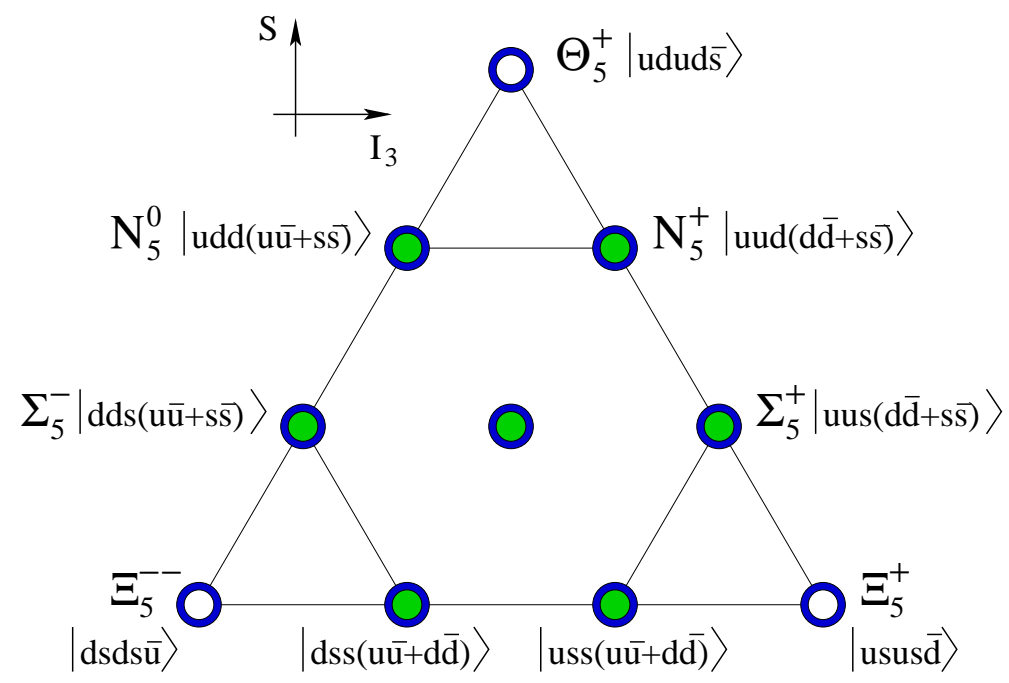

Figure 7: Light pentaquark anti-decuplet and octet.

\section{Pentaquark searches at BABAR}

In the past two years, several experiments reported evidence for new baryon states that are compatible with being bound states of five quarks (pentaquarks). The most convincing evidence is for an exotic $(B=1, S=1)$, light resonance called $\Theta^{+}(1540)$ [24-30]. This state has an unusually narrow width for a particle that could decay strongly. Recently, NA49 published evidence for two heavier narrow states $\left(\Xi^{--}\right.$and $\left.\Xi^{0}\right)$ [31], while H1 observed a narrow exotic charmed resonance $\left(\Theta_{c}^{0}\right)$ with mass of $3099 \mathrm{MeV} / c^{2}[32]$.

Several theoretical models have been proposed to describe the pentaquark structure [33-35]. The prediction is that the lowest mass states containing $u, d$ and $s$ quarks should occupy a spin- $\frac{1}{2}$ anti-decuplet and octet as illustrated in figure 7

Although experiments with baryon beams may have an advantage in producing these states, we know that $e^{+} e^{-}$collisions produce quite democratically all of the other particles. It has been experimentally observed that for baryons with non-zero $B, C$ or strangeness, and/or orbital angular momentum, the production rates seem to depend only on mass and spin, and not on the quark content. If pentaquarks are produced similarly, their production rate should be as high as that for ordinary baryons of the same mass and spin, i.e., about $8 \times 10^{-4}$ for the $\Theta_{5}^{+}(1540)$ and $4 \times 10^{-5}$ for the $\Theta_{5}^{--}[36]$.

It has been recently suggested that heavier pentaquarks such as the $\Theta^{*++}$ could be accessible at the B factories. The $\Theta^{*++}$ is a member of the baryon 27-plet containing the quarks uuuds and has $I=1$ and $I_{3}=1$. It is predicted to decay into the final state $p K^{+}$, and to have a mass between 1.43 and $1.70 \mathrm{GeV} / c^{2}$ with a width of $37-80 \mathrm{MeV} / c^{2}$ [37].

In this section I discuss the results of several searches for light [38] and heavy [39] pentaquarks recently published by BABAR. Light strange pentaquarks belonging to the decuplet and octet represented in figure 7 are inclusively searched for in $123 \mathrm{fb}^{-1}$. The analysis focuses on final states containing strange particles and protons that are easily identifiable in the detector. The search for the $\Theta^{*++}$ follows a very different approach, involving the exclusive reconstruction of $B^{+}$mesons decaying into $p \bar{p} K$ final states, as suggested in [40]. This analysis is performed with $81 \mathrm{fb}^{-1}$. 

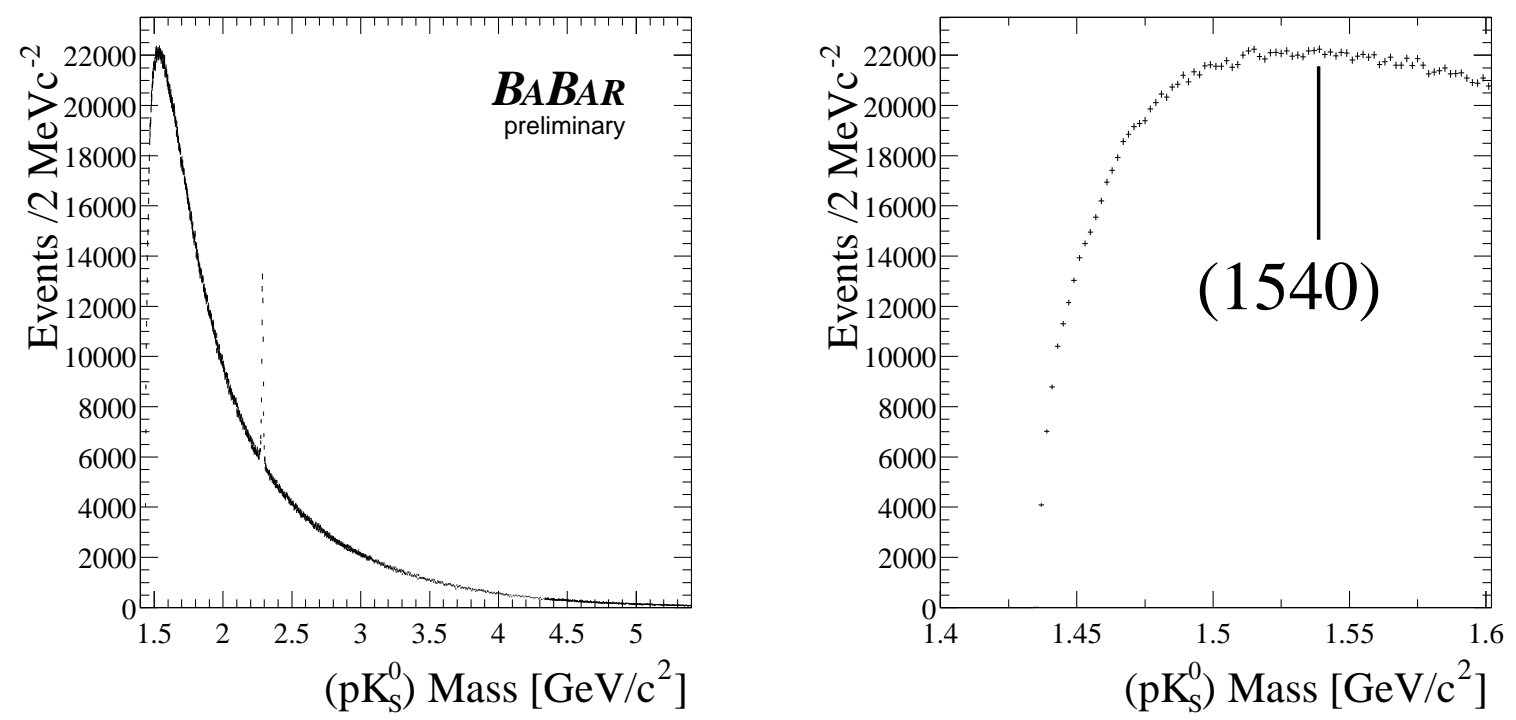

Figure 8: $p K_{S}^{0}$ invariant mass distributions in the $\Theta_{5}^{+}(1540)$ search.

\subsection{Search for $\Theta_{5}^{+}(1540) \rightarrow p K_{S}^{0}$}

This analysis aims to reconstruct decays of the $\Theta_{5}^{+}(1540)$ into the $p K_{S}^{0}$ final state. The $K_{S}^{0}$ candidates are reconstructed in their decay into two charged pions, with selection criteria designed to avoid a bias toward any specific production mechanism, while keeping a high reconstruction efficiency. The $K_{S}^{0}$ candidates are then combined with protons from the interaction point to form the $\Theta_{5}^{+}(1540)$ candidates.

The invariant mass distribution of the $p K_{S}^{0}$ pairs is shown in figure 8 . The clear peak around $2285 \mathrm{MeV} / c^{2}$ visible on the left plot is due to the decay $\Lambda_{c}^{+} \rightarrow p K_{S}^{0}$. The abundant signal and the narrow peak demonstrate our sensitivity to the presence of a narrow resonance. The left plot of figure 8 is a magnified view of the region where the $\Theta_{5}^{+}(1540)$ is expected. No signal is observed. Null results are obtained also when the search is performed separately for ten momentum bins uniformly distributed between zero and $5 \mathrm{GeV} / c$.

The null results are used to set a limit on the differential production cross section of $\Theta_{5}^{+}(1540)$ in $e^{+} e^{-}$interaction. In the calculation the mass is assumed to be $1540 \mathrm{MeV} / c^{2}$. Since the natural width of this particle is unknown, two hypotheses have been considered: $\Gamma=8 \mathrm{MeV} / c^{2}$, corresponding to the experimental upper limit, and $\Gamma=1 \mathrm{MeV} / c^{2}$, corresponding to a very narrow state. The $95 \%$ C.L. upper limit on the differential production cross section of the $\Theta_{5}^{+}(1540)$ is shown in figure 9. The branching fraction $\mathcal{B}\left(\Theta_{5}^{+}(1540) \rightarrow p K_{S}^{0}\right)$ is assumed to be $1 / 4$. Taking the upper limit width, the $95 \%$ C.L. upper limit on the total production rate is calculated to be $1.1 \times 10^{-4}$ per $q \bar{q}$ event, roughly a factor of eight below the values measured for ordinary baryons of similar mass as shown in figure 10.

\subsection{Search for $\Xi_{5}^{--} \rightarrow \Xi^{-} \pi^{-}$and $\Xi_{5}^{0} \rightarrow \Xi^{-} \pi^{+}$}

The $\Xi_{5}^{--}$and $\Xi_{5}^{0}$ states were searched for in decays involving $\Xi^{-}$baryon and a charged pion. The $\Xi^{-}$is reconstructed in its decay $\Xi^{-} \rightarrow \Lambda^{0} \pi^{-}$with $\Lambda^{0} \rightarrow p \pi^{-}$. As for the $\Theta_{5}^{+}(1540)$ search, all 

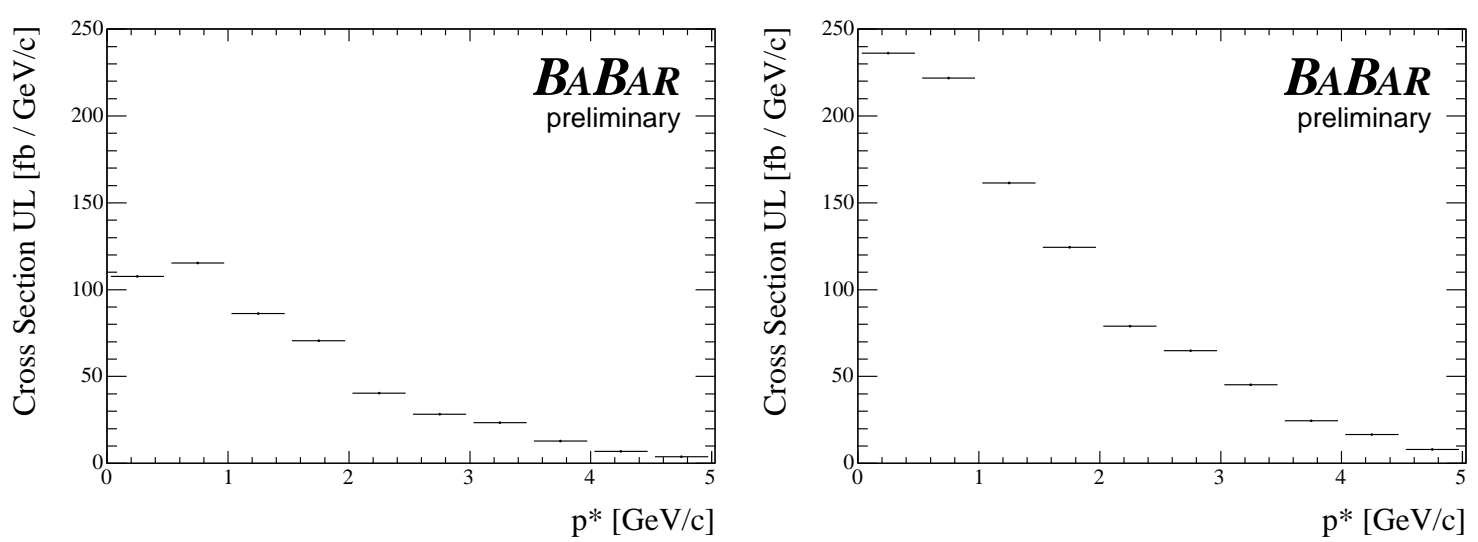

Figure 9: 95\% C.L. upper limit of the differential production cross section of $e^{+} e^{-} \rightarrow \Theta_{5}^{+}(1540) X$, assuming $\Gamma=8 \mathrm{MeV} / c^{2}$ (left) and $1 \mathrm{MeV} / c^{2}$ (right).

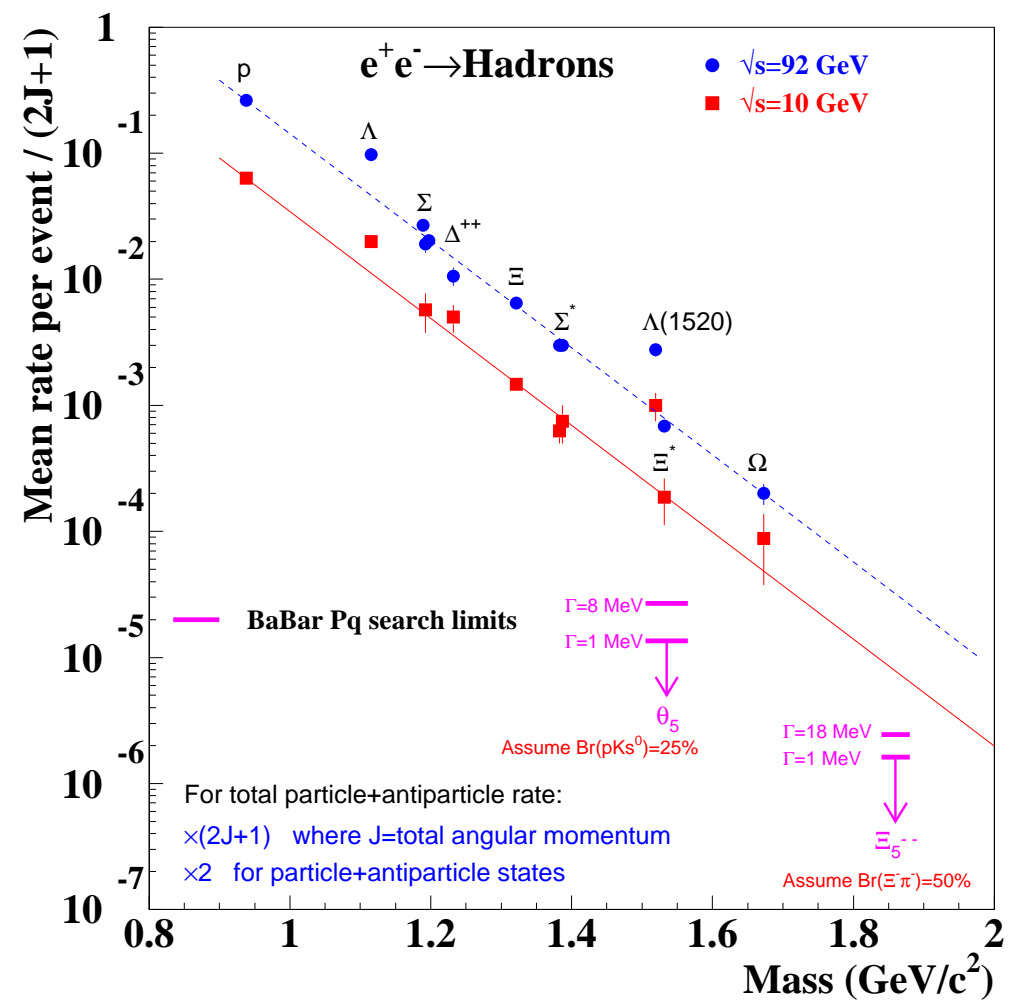

Figure 10: Baryon production rates in $e^{+} e^{-}$interactions [36] measured at the $Z^{0}$ pole (circles) and $\Upsilon(4 S)$ (squares). The vertical axis accounts for number of spin and particle+antiparticle states. The lines are chosen to guide the eye. The arrows indicate the upper limits on the $\Theta_{5}^{+}$and $\Xi_{5}^{--}$. 

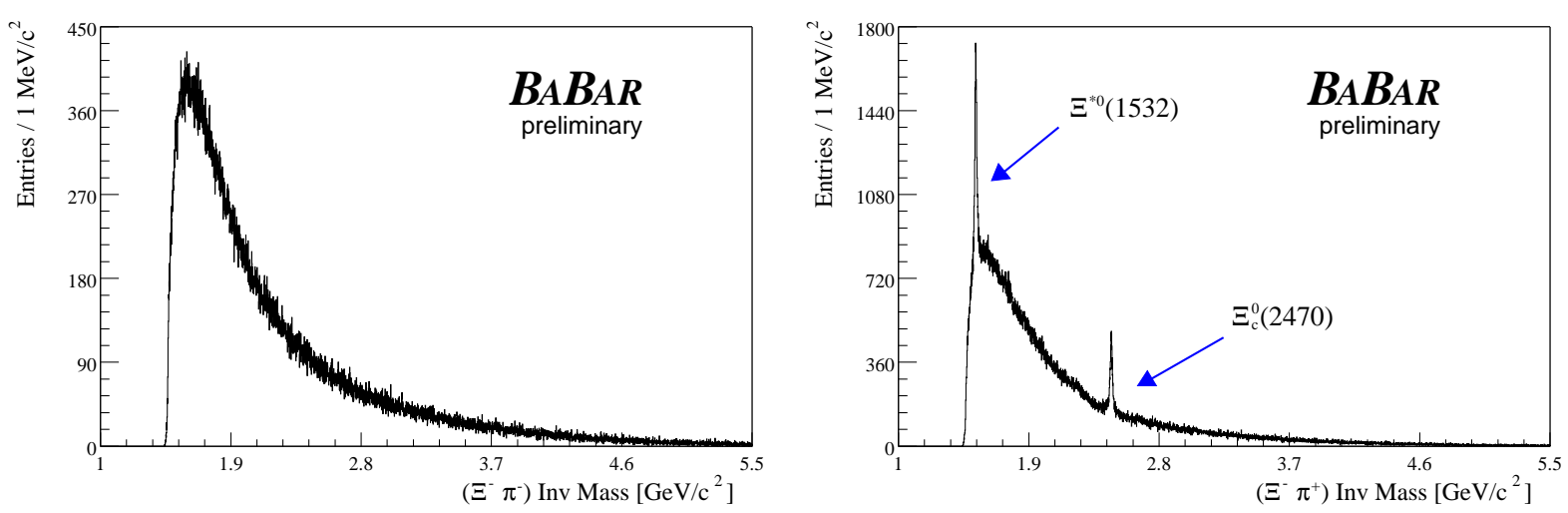

Figure 11: $\Xi^{-} \pi^{-}$(left) and $\Xi^{-} \pi^{+}$(right) invariant mass distributions in the $\Xi_{5}^{--}$and $\Xi_{5}^{0}$ searches.

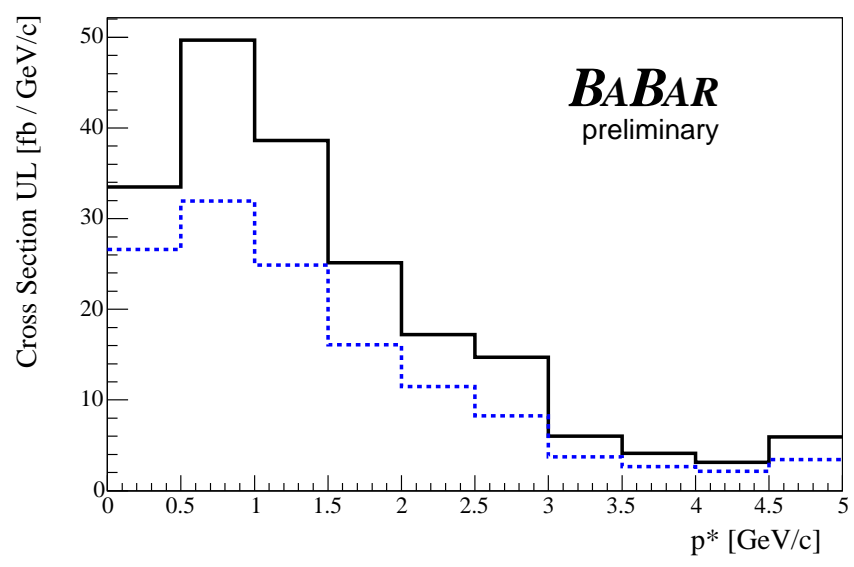

Figure 12: 95\% C.L. upper limit of the differential production cross section of $e^{+} e^{-} \rightarrow \Xi_{5}^{--} X$, assuming $\Gamma=18 \mathrm{MeV} / c^{2}$ (solid line) and $1 \mathrm{MeV} / c^{2}$ (dotted line).

selection criteria used in the analysis are designed to maximize the efficiency and avoid a bias toward any specific production mechanism.

The invariant mass distributions for the $\Xi^{-} \pi^{-}$and $\Xi^{-} \pi^{+}$pairs are illustrated in figure [1]. No resonance is visible in the $\Xi^{-} \pi^{-}$invariant mass distribution. Two peaks are instead visible in the $\Xi^{-} \pi^{+}$plot, corresponding to the decays of $\Xi^{* 0}(1532)$ and $\Xi_{c}^{0}(2470)$, but no structure is visible in the region where the $\Xi_{5}^{0}$ is expected.

The absence of a $\Xi_{5}^{--}$signal is reflected as an upper limit at the $95 \%$ C.L. on the differential production cross section shown in figure 12 as a function of $p^{*}$, the momentum in the $e^{+} e^{-}$center of mass frame. The branching fraction $\mathcal{B}\left(\Xi_{5}^{--} \rightarrow \Xi^{-} \pi^{-}\right)$is assumed to be $1 / 2$. Assuming a width of $18 \mathrm{MeV} / c^{2}$, the $95 \%$ C.L. upper limit on the total production rate is calculated to be $1.0 \times 10^{-5}$ per $q \bar{q}$ event, roughly a factor of two below the values measured for ordinary baryon of similar mass as illustrated in figure 10. 


\subsection{Inclusive searches for other strange pentaquarks}

BABAR also performs a more inclusive search for other strange pentaquarks decaying into the final states $\Lambda^{0} K$ and $\Sigma^{0} K$, where $K$ is either a charged or a neutral kaon and $\Sigma^{0} \rightarrow \Lambda^{0} \gamma$ and $\Lambda^{0} \rightarrow p \pi^{-}$. These final states give access to the pentaquarks $\Xi_{5}^{-}, \Xi_{5}^{0}, N_{5}^{0}$ and $N_{5}^{+}$introduced in figure 7 Inclusive searches are also performed for $\Sigma_{5}^{+} \rightarrow p K_{S}^{0}$. No pentaquark signal is observed in any of these inclusive searches.

\subsection{Search for the $\Theta^{*++}$ pentaquark in exclusive B decays}

In this analysis, the $B^{+}$mesons are exclusively reconstructed in the $p \bar{p} K^{+}$final state. The purity of the signal is increased by cuts on the invariant mass and energy of the $B$ candidate, and by the use of topological variables that distinguish continuum from $B \bar{B}$ events. Of the 212 candidates reconstructed in this analysis, $188 \pm 17$ are estimated to be real $B^{+}$decays, including $68 \pm 10$ containing charmonium decays to $p \bar{p}$.

The $p K^{+}$invariant mass distribution of the selected $B$ candidates is analyzed searching for a pentaquark signal up to $2.36 \mathrm{GeV} / c^{2}$. No events are observed below $1.85 \mathrm{GeV} / c^{2}$, and no structure is visible in the entire area studied. The $95 \%$ C.L. upper limit for the branching fraction of $B^{+} \rightarrow \Theta^{*++}\left(p K^{+}\right) \bar{p}$ is measured to be $1.5 \times 10^{-7}$ for $1.43<m\left(\Theta^{*++}\right)<1.85 \mathrm{GeV} / c^{2}, 2.4 \times 10^{-7}$ for $1.85<m\left(\Theta^{*++}\right)<2.00 \mathrm{GeV} / c^{2}$, and $3.3 \times 10^{-7}$ for $2.00<m\left(\Theta^{*++}\right)<2.36 \mathrm{GeV} / c^{2}$.

\section{Summary}

Many new results in the field of hadronic physics were recently published by the BABAR Collaboration.

The discovery of the $D_{s J}^{*}(2317)^{+}$narrow state revived the interest in charm spectroscopy. The mass of the $D_{s J}^{*}(2317)^{+}$and of its heavier twin, the $D_{s J}(2460)^{+}$, are now measured with high precision in many different decay modes. In addition, the measurement of other properties, such as branching fractions and angular distributions, are now accessible, thanks to the pure samples of $D_{s J}$ mesons reconstructed in $B$ decays.

The narrow state $X(3872)$ has recently been observed by BABAR, in agreement with previous results from Belle. The $D_{s J}(2632)$ state reported by SELEX, on the other hand, has not been confirmed.

BABAR also searches for pentaquark production in $e^{+} e^{-}$interactions. Various states are investigated, and particular attention is given to the searches for the $\Theta_{5}^{+}(1540)$ and the $\Xi^{--}(1860)$. The null results reflect limits on the rates of the production of such states in $e^{+} e^{-}$events, well below the rates measured for ordinary baryons of similar masses.

\section{Acknowledgments}

It is a pleasure to thank my BABAR colleagues P. Burchat, V. Halyo, V. Luth, M. Morii and R. Yamamoto for helpful comments. I would also like to thank the organizers of the APS Topical Group on Hadronic Physics for creating such a pleasant and productive environment. This work was supported by the DOE contracts DE-FC02-94ER40818 and DE-AC02-76SF00515. 


\section{References}

[1] B. Aubert et al. [BABAR Collaboration], Nucl. Instr. Meth. A479, 1 (2002).

[2] B. Aubert et al. [BABAR Collaboration], Phys. Rev. Lett. 90, 242001 (2003).

[3] D. Besson et al. [CLEO Collaboration], Phys. Rev. D 68, 032002 (2003).

[4] B. Aubert et al. [BABAR Collaboration], Phys. Rev. D 69, 031101 (2004).

[5] K. Abe et al. [Belle Collaboration], Phys. Rev. Lett. 92, 012002 (2004).

[6] R. N. Cahn and J. D. Jackson, Phys. Rev. D 68, 037502 (2003).

[7] W. A. Bardeen, E. J. Eichten and C. T. Hill, Phys. Rev. D 68, 054024 (2003).

[8] Y. B. Dai, C. S. Huang, C. Liu and S. L. Zhu, Phys. Rev. D 68, 114011 (2003).

[9] A. Deandrea, G. Nardulli and A. D. Polosa, Phys. Rev. D 68, 097501 (2003).

[10] T. Barnes, F. E. Close and H. J. Lipkin, Phys. Rev. D 68, 054006 (2003).

[11] E. van Beveren and G. Rupp, Phys. Rev. Lett. 91, 012003 (2003).

[12] H. Y. Cheng and W. S. Hou, Phys. Lett. B 566, 193 (2003).

[13] A. P. Szczepaniak, Phys. Lett. B 567, 23 (2003).

[14] T. E. Browder, S. Pakvasa and A. A. Petrov, Phys. Lett. B 578, 365 (2004).

[15] B. Aubert et al. [BABAR Collaboration], Phys. Rev. Lett. 93, 181801 (2004).

[16] B. Aubert et al. [BABAR Collaboration], arXiv:hep-ex/0408067.

[17] B. Aubert et al. [BABAR Collaboration], arXiv:hep-ex/0406022.

[18] B. Aubert et al. [BABAR Collaboration], arXiv:hep-ex/0408087.

[19] S. K. Choi et al. [Belle Collaboration], Phys. Rev. Lett. 91, 262001 (2003).

[20] D. Acosta et al. [CDF II Collaboration], Phys. Rev. Lett. 93, 072001 (2004).

[21] E. J. Eichten, K. Lane and C. Quigg, Phys. Rev. Lett. 89, 162002 (2002); E. J. Eichten, K. Lane and C. Quigg, Phys. Rev. D 69, 094019 (2004).

[22] S. Pakvasa and M. Suzuki, Phys. Lett. B 579, 67 (2004); N. A. Tornqvist, arXiv:hep-ph/0308277; F. E. Close and P. R. Page, Phys. Lett. B 578, 119 (2004); E. S. Swanson, Phys. Lett. B 588, 189 (2004); T. Barnes and S. Godfrey, Phys. Rev. D 69, 054008 (2004); M. B. Voloshin, Phys. Lett. B 579, 316 (2004).

[23] A. V. Evdokimov et al. [SELEX Collaboration], Phys. Rev. Lett. 93, 242001 (2004).

[24] T. Nakano et al. [LEPS Collaboration], Phys. Rev. Lett. 91, 012002 (2003).

[25] J. Barth et al. [SAPHIR Collaboration], Phys. Lett. B 572, 127 (2003). 
[26] V. Kubarovsky et al. [CLAS Collaboration], Phys. Rev. Lett. 92, 032001 (2004).

[27] V. V. Barmin et al. [DIANA Collaboration], Phys. Atom. Nucl. 66, 1715 (2003)

[28] A. Aleev et al. [SVD Collaboration], arXiv:hep-ex/0401024.

[29] A. Airapetian et al. [HERMES Collaboration], Phys. Lett. B 585, 213 (2004).

[30] M. Abdel-Bary et al. [COSY-TOF Collaboration], Phys. Lett. B 595, 127 (2004).

[31] C. Alt et al. [NA49 Collaboration], Phys. Rev. Lett. 92, 042003 (2004).

[32] A. Aktas et al. [H1 Collaboration], Phys. Lett. B 588, 17 (2004).

[33] D. Diakonov, V. Petrov and M. V. Polyakov, Z. Phys. A 359, 305 (1997).

[34] M. Karliner and H. J. Lipkin, Phys. Lett. B 575, 249 (2003).

[35] R. L. Jaffe and F. Wilczek, Phys. Rev. Lett. 91, 232003 (2003).

[36] K. Hagiwara et al. [Particle Data Group], Phys. Rev. D 66, 010001 (2002).

[37] B. Wu and B. Q. Ma, Phys. Rev. D 69, 077501 (2004); H. Walliser and V. B. Kopeliovich, J. Exp. Theor. Phys. 97, 433 (2003) [Zh. Eksp. Teor. Fiz. 124, 483 (2003)]; D. Borisyuk, M. Faber and A. Kobushkin, arXiv:hep-ph/0307370

[38] B. Aubert et al. [BABAR Collaboration], arXiv:hep-ex/0408064.

[39] B. Aubert et al. [BABAR Collaboration], arXiv:hep-ex/0408037.

[40] T. E. Browder, I. R. Klebanov and D. R. Marlow, Phys. Lett. B 587, 62 (2004). 


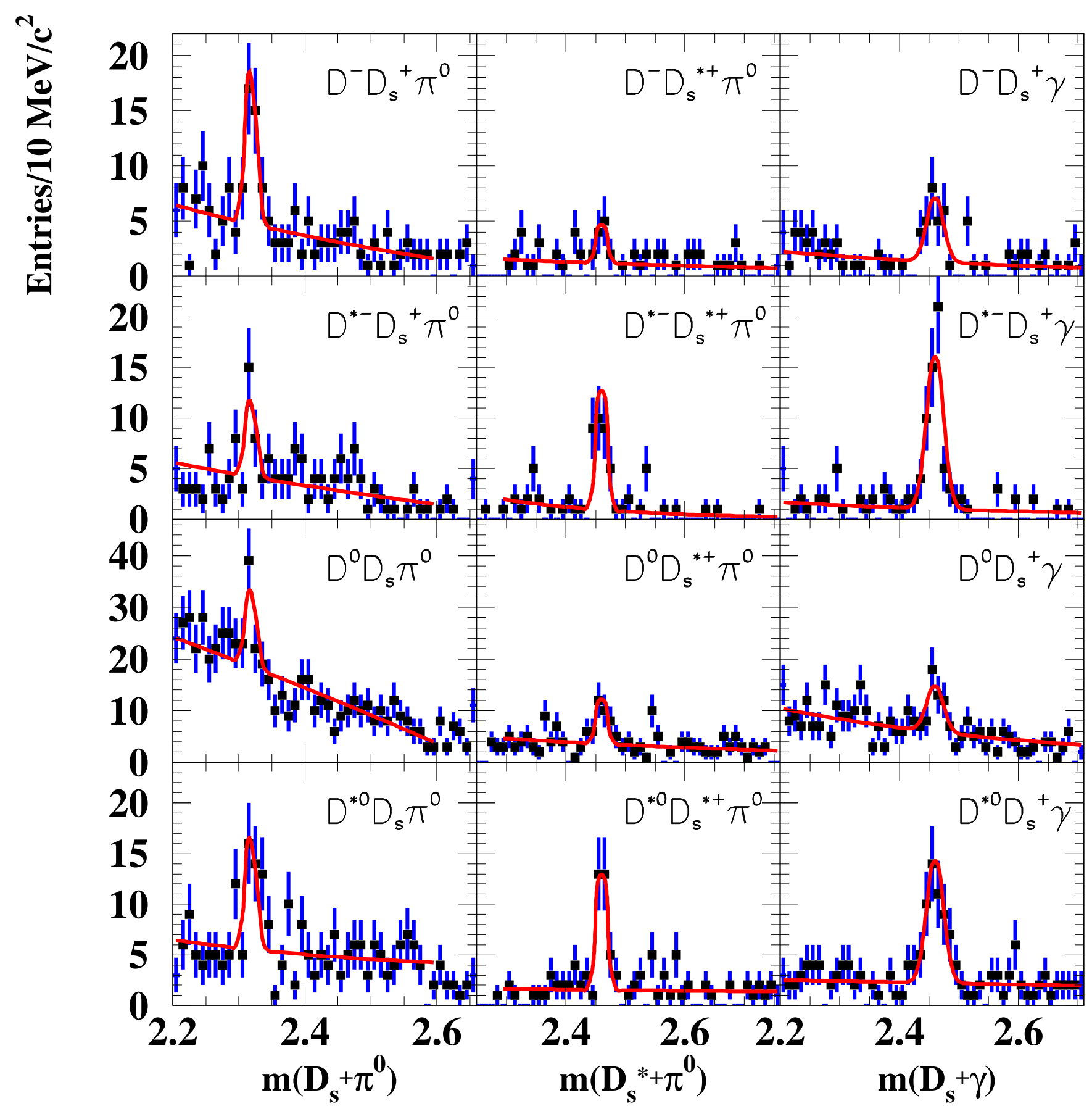

